\title{
"Twins Intramedullary Tumors": Case Report of a Double Spinal Metastasis from Breast Cancer. Technical Nuances and Review of the Literature
}

\author{
Pizzuti $V^{1,2 *}$, Ricciardi F ${ }^{1}$, D Elia $A^{1}$, Fabbiano $F^{1}$ and Innocenzi $G^{1}$ \\ ${ }^{1}$ Department of Neurosurgery, I.R.C.C.S. Neuromed Pozzilli (IS), Via Atinense 18 Italy \\ ${ }^{2}$ Department of Neurology and Psichiatry, "La Sapienza” University, Rome, Italy
}

*Corresponding author: Pizzuti V, Department of Neurosurgery, I.R.C.C.S. Neuromed Pozzilli (IS), Via Atinense 18 Italy and Department of Neurology and Psichiatry, "La Sapienza" University, Rome, Italy

\section{ARTICLE INFO}

Received: 幽 July 08, 2020

Published: 慧 July 23, 2020

Citation: Pizzuti V, Ricciardi F, D Elia A, Fabbiano F, Innocenzi G. "Twins Intramedullary Tumors": Case Report of a Double Spinal Metastasis from Breast Cancer. Technical Nuances and Review of the Literature. Biomed J Sci \& Tech Res 29(1)-2020. BJSTR. MS.ID.004749.

Keywords: Intramedullary Spinal Cord Metastases (ISCM); Intraoperative Neurophysiological Monitoring (IONM); Breast Cancer; Twins Lesions

\section{ABSTRACT}

Objective: Intramedullary spinal cord metastases (ISCM) from breast cancer are very rare. These patients show rapidly progressive neurological deficits and require immediate examination. The aim of surgery is decompression of neural tissue and to obtain a histological evaluation of the tumor. When ISCM occur, they are usually solitary. In this paper, we present the unique case of two distinct breast cancer metastases in the cervical spinal intramedullary area, occurring simultaneously.

Case: A 46-year-old woman with breast cancer diagnosed 1 year earlier was admitted to our hospital with insidious onset of tetraparesis and neck pain, associated with sensory disturbances in the left hemibody. Seven months earlier, she had been surgically treated for a solitary cervical intramedullary metastasis from breast carcinoma. Cervical Magnetic Resonance Imaging (MRI) at admission revealed two gadolinium enhancing intramedullary lesions, that we surgically removed under intraoperative neurophysiological monitoring (IONM). Pathological examination of the lesion was again consistent with breast cancer metastases.

Conclusion: ISCMs from breast cancer are rare and usually solitary. To our knowledge, this is the first case reported in the literature of two adjacent simultaneous intramedullary lesions. Clinical and radiological features are reported, and the pertinent literature is reviewed.

\section{Introduction}

Intramedullary spinal cord metastases (ISCMs) represent a rare complication of malignancies and have been identified in 0.9$2.1 \%$ of all autopsy cases of cancer [1-3]. Lung cancer and breast carcinoma represent the majority of primary tumors $(59 \%$ and $14 \%$, respectively) [4], and the reported incidence is estimated at $0.1-0.4 \%[4,5]$. They become clinically evident in less than $1 \%$ of patients suffering from metastatic disease [6,7]. Most of them present with rapid progressive neurological deficits and require immediate evaluation $[2,3,6,8,9]$. Nowadays, spinal magnetic resonance imaging (MRI) is routinely applied for diagnosis. Surgery aims at decompression of functional neural tissue and histological confirmation of the tumor [9]. Metastatic localization of a malignant disease is usually solitary and at clinical onset the patient has systemic widespread with secondary localization to lung, liver, bones and brain [10]. In this report we describe the case of a 49 year-old woman, affected by breast cancer, we have operated on for two adjacent cervical ISCMs. To our knowledge, this is the first surgical case reported in literature of a double intramedullary metastatic disease, with the two separate lesions occurring simultaneously. The aim of our paper is to describe the clinical features and radiological findings of metastatic intramedullary breast carcinoma, as well as surgical technique with emphasis on intraoperative neurophysiological monitoring (IONM) and a wide literature review about ISCMs from breast cancer. 


\section{Case Report}

A 49-year-old female patient, who had undergone radical mastectomy for ductal carcinoma 1 year before, was admitted to our Institution in June 2016. She presented with left hemiparesis and neck pain associated with hypoesthesia below the C6 dermatome in upper and lower limbs, worse on the left side. Lhermitte sign was positive. Cervical MRI clearly showed a solid mass, occupying almost the entire spinal cord from $\mathrm{C} 5$ to $\mathrm{C} 6$, well circumscribed and associated to homogeneous contrast enhancement and peritumoral edema from C1 to C8 (Figure 1). Her past medical history was significant for breast tumor diagnosed as ductal carcinoma 1 year earlier. At the time of presentation, there was no evidence of metastases on Whole Body Computer Tomography (WBCT).

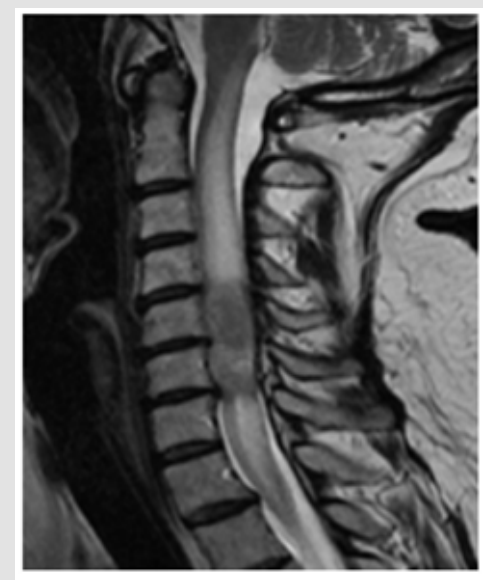

Figure 1a

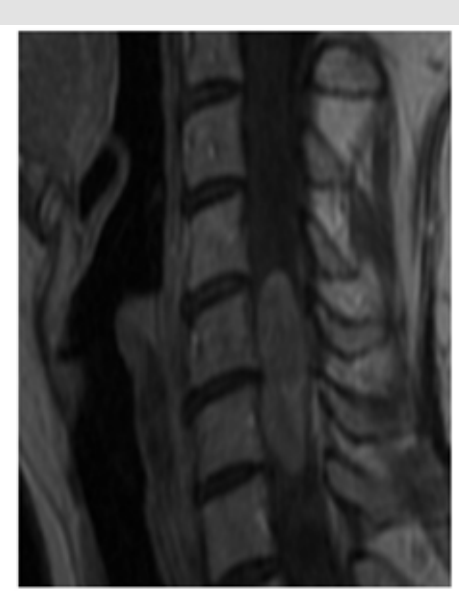

Figure $1 b$

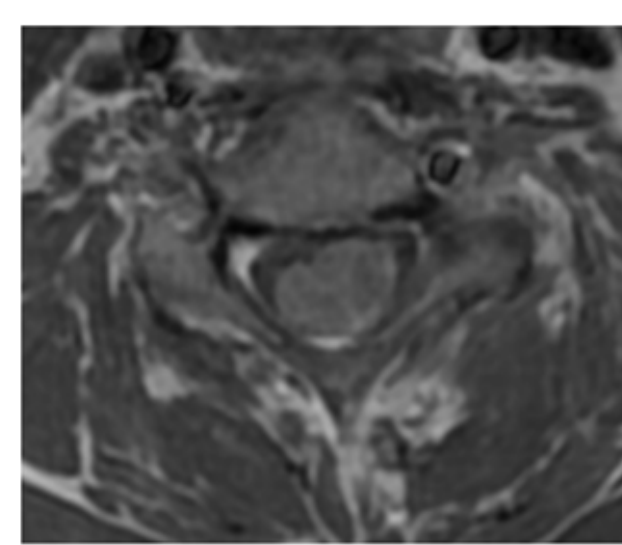

Figure 1c

Figure 1: Sagittal (1a and 1b) and axial (1c) images of an isointense mass lesion with intramedullary edema from C1 to C8 (1a). Intramedullary tumor extending from C5 to C6 (1b), occupying almost entirely the spinal cord (1c), well circumscribed and with homogeneus contrast enhancement.

Surgery was performed after induction of general endotracheal anesthesia and with the assistance of intraoperative neurophysiological monitoring of Motor Evocked Potentials (MEPs) and Somatosensory Evocked Potentials (SSEPs), with the patient in a prone position. A median longitudinal skin incision was performed from the inion to $\mathrm{C} 7$ and, after bilateral exposure of the cervical spine, laminectomy of C4, C5 and C6 was executed. With the assistance of the operating microscope, the dura was opened, and the tumor was visualized: intraoperative Ultrasound (US) was used at this stage to check both the cranial and caudal extremities of the lesion. The tumor was bloody and infiltrative, without a clear cleavage plane. However, gross total removal (GTR) was achieved using bipolar forceps and ultrasound aspirator. Intraoperative neurophysiological parameters remained unchanged throughout the procedure. Hemostasis of the operative field was achieved, and the dura, cervical fascia, subcutaneous tissue and skin were sutured separately. Post-operative cervical MRI showed radical removal and disappearance of peritumoral edema (Figure 2). Soon after the operation, patient's hemiparesis deteriorated (strength 2/5 according to Medical Research Council, MRC scale), but it progressively improved after one week, also with the aid of physical therapy. Histological examination revealed a ductal carcinoma with cytocheratin expression and positivity for Estrogen and Progesterone receptors (ER+, PR+).

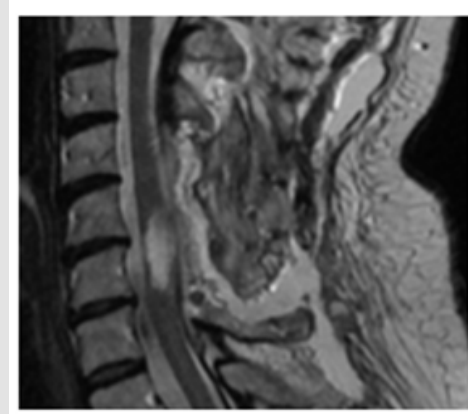

Figure 2a

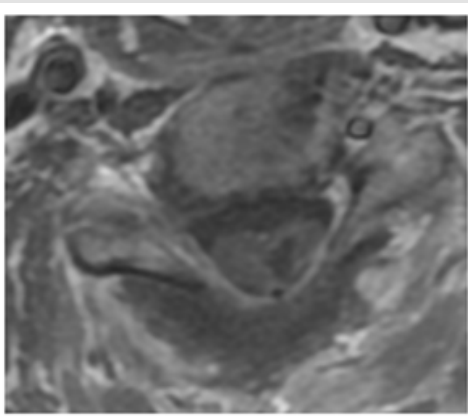

Figure 2b

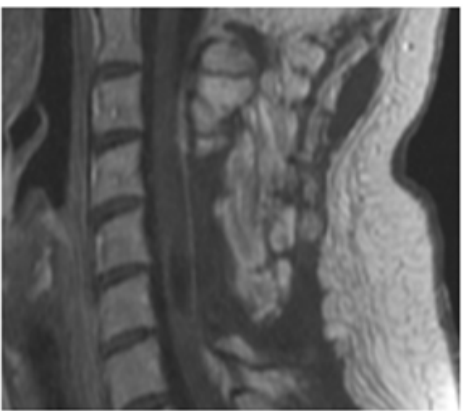

Figure 2c

Figure 2: Postoperative T2 sequences showing that intramedullary edema disappeared (2a). T1 post-gadolinium sequences showing gross total removal of the intramedullary tumor ( $2 \mathrm{~b}$ and $2 \mathrm{c})$. 
Post-operative period was uneventful for seven months, after which the patient presented once again with progressive neurological impairment. On her physical examination, she showed spastic tetraparesis that was more severe in the lower limbs $(3 / 5$ according to the MRC scale) than in the upper ones (4/5 according to the MRC scale), with lower limbs hyperreflexia, while biceps and triceps reflexes were reduced. She also complained about pain and temperature sensory loss in the left hemibody, where she also felt electric shock-like dysesthesias. Cervical MRI with gadolinium showed at C5 level a solid mass (maximum diameter $3 \mathrm{~mm}$ ), characterized by homogeneous contrast enhancement, associated to another tumoral lesion (maximum diameter $11 \mathrm{~mm}$ ) with the same radiological features (Figures $3 \& 4$ ). WBCT scan was, again, normal.

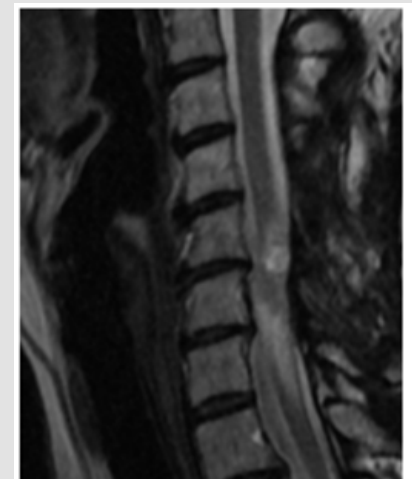

Figure 3a

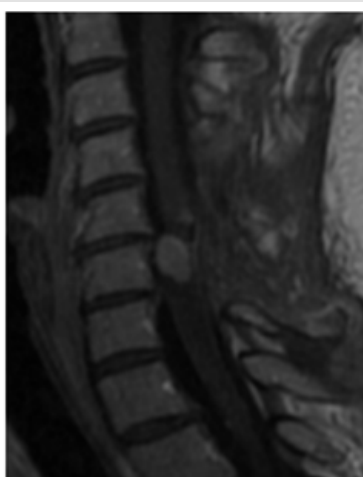

Figure 3b

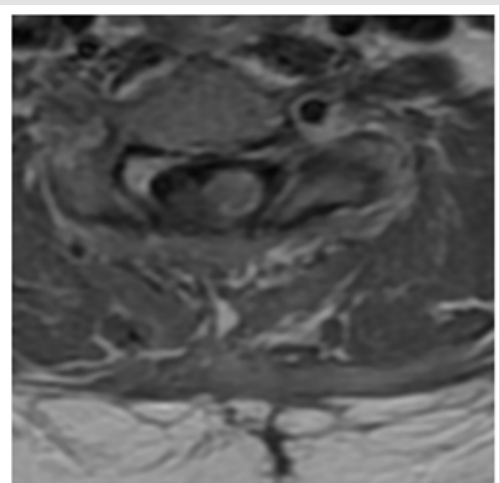

Figure 3c

Figure 3: Cervical contrast-enhanced MRI showing two solid masses at C5 and C6 levels. 3a and 3b: sagittal views; 3c: axial view.

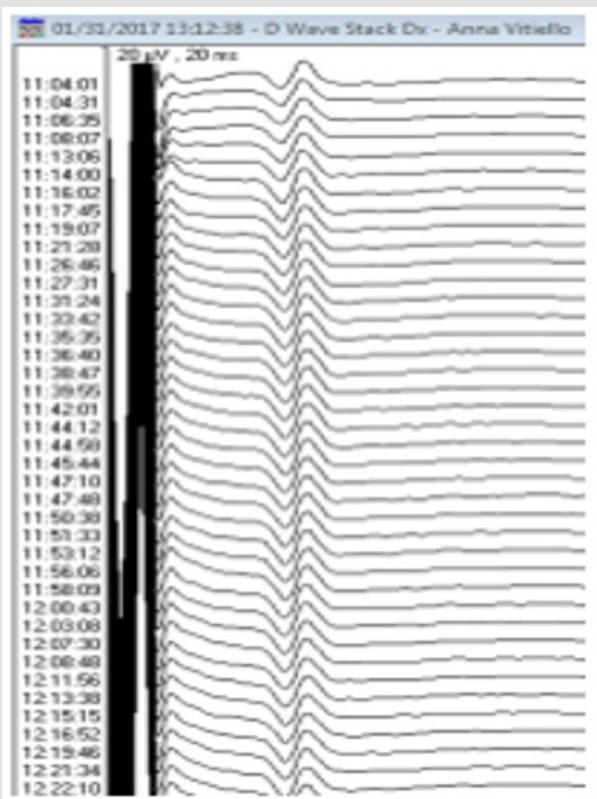

Figure 4a

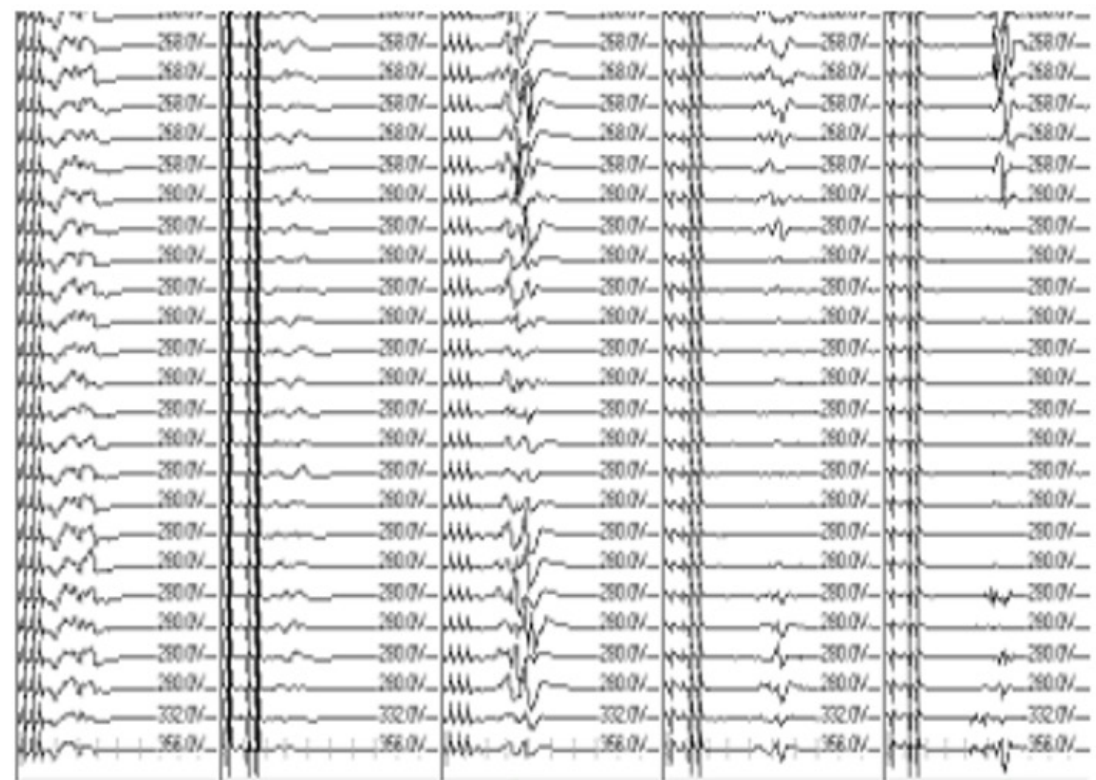

Figure $4 b$

Figure 4: IONM during surgical procedure. IONM during surgical procedure with MEPs that disappeared during Ultrasonic Aspirator utilization (A) being at the same time D-Wave present (B).

The patient underwent the second surgical procedure using intraoperative neurophysiological monitoring of MEPs, SSEPs and D Wave. Once the previously performed skin incision was opened, tumoral lesion at $\mathrm{C} 5$ level was removed en bloc without any change in MEPs, SSEPs and D-wave. In contrast, the twin lesion at C6 level was firmer and more adherent to the spinal cord parenchyma, thus requiring the use of ultrasonic aspirator (Sonoca 300, power 30, irrigation 30, aspiration 20) to obtain radical removal. During this second surgical step, a transient disappearance of MEP of anterior tibialis and wrist abduttor in the left leg was observed, while D 
wave remained stable. At the end of the surgical procedure, MEPs returned to normal values. In fact, strength in upper and lower limbs progressively improved following the operation, as well as patient's painful sympthoms. Cervical MRI with gadolinium performed on the fifth post-operative day showed complete resection and an area of contrast enhancement at C5-C6 level, as in keeping with surgery-related artifacts (Figure 5). At discharge, she was able to stand upright and walk without assistance. Histological examination confirmed the previous diagnosis: ductal carcinoma, with cytocheratin expression, ER+, PR+ and with Ki 67 5-6\% (Figure 6).
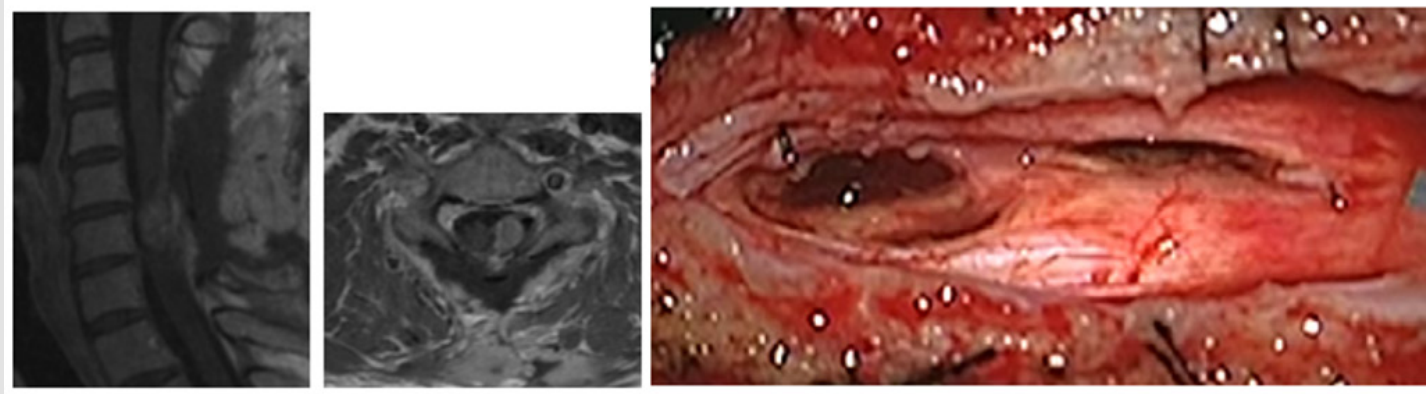

Figure 5

Figure 5: Cervical contrast enhanced MRI performed after the second surgical procedure showing contrast enhancement (a) and operatory field after that surgical removal was achieved (b).
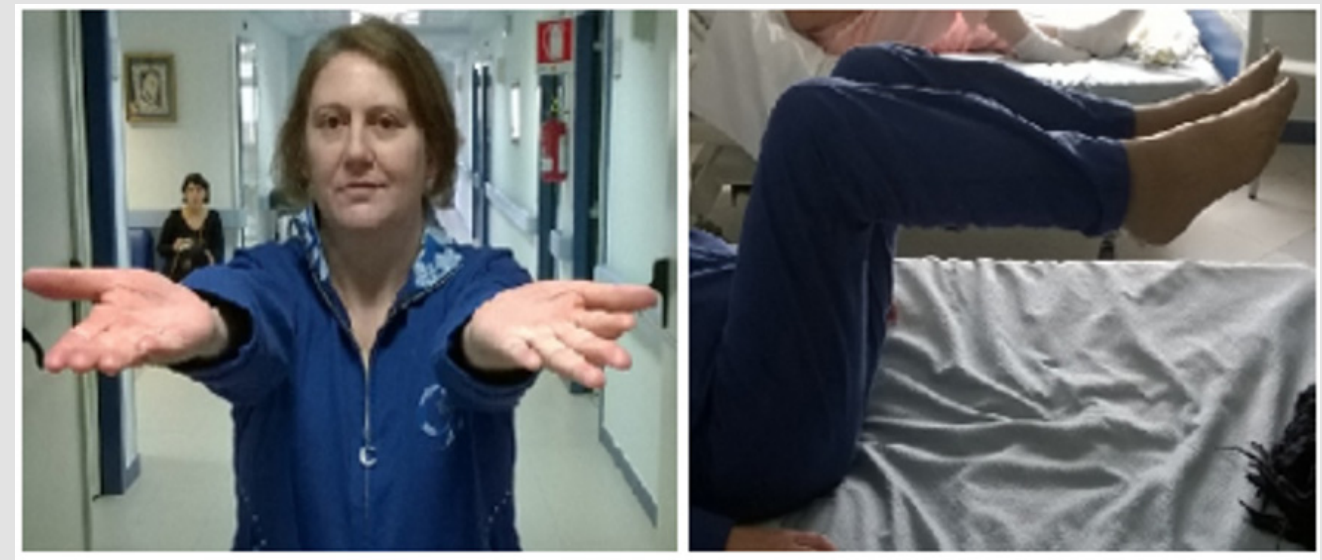

Figure 6

Figure 6: Neurological patient's status after the second surgical procedure.

\section{Discussion}

Intramedullary spinal cord metastases represent a small proportion of intramedullary tumors, accounting for $0.9-5 \%$ of all spinal metastases $[3,6,11]$. Development of an ISCM is still a rare event in the course of a malignant disease. Nevertheless, due to both increasing survival of patients with malignant tumors and widespread MRI availability, incidence of ISCM is rising $[4,5,11]$. Three different routes for the spread of tumor to the spinal cord have been hypothesized, among which the hematogenous dissemination through the arterial route is considered to be the most common one $[2,3,6-8,12-15]$. The second mechanism is related to the leptomeningeal dissemination by the cerebrospinal fluid (CSF) $[3,6,14]$. Thirdly, direct invasion by a metastatic tumor from the spinal extradural space or CSF or nerve roots, through the dura and into the cord, and spread to the subarachnoid space and spinal cord parenchyma, has been suggested $[14,16]$.

ISCMs are often asymptomatic, and clinically affect less than $1 \%$ of patients with metastatic cancer $[5,6,17]$. At time of presentation, weakness is present in $93 \%$, sensory loss in $78 \%$ and urogenital dysfunction in $62 \%$ of patients $[4,7,10,13,18]$. Rapid progression of the symptoms distinguishes ISCMs from primary intramedullary neoplasms, which are typically characterized by a slower progression $[10,13]$. At the time of diagnosis, most of the patients have systemic metastases to lungs, liver and bones, and nearly half of them have brain metastases [7,12,17]. For this reason, imaging modalities should be extended to the entire spinal cord and brain, and the whole body needs to be investigated to detect any further dissemination. Prompt and accurate diagnosis is crucial for 
ISCM effective treatment, and spinal MRI is the imaging technique of choice [17], especially T2-weighted images are highly sensitive in the detection of spinal cord edema $[4,7,12]$. The two most pertinent imaging features are: intramedullary enhancing mass lesion; extensive (for lesion size, 3 or more levels) T2 spinal cord hyperintensity, representing edema. On the other hand, presence of cystic changes/hemorrhage makes ISCM diagnosis unlikely [19].

Optimum treatment for ISCMs remains controversial due to the lack of controlled studies on the results of different therapeutic options [17]. However, three treatment modalities are available for ISCM: radiotherapy, chemotherapy, and surgery. Thanks to the increased accuracy of diagnostic tools and developments in microsurgical techniques, surgery has become a viable treatment option in a rising number of ISCMs cases $[8,16,20]$. It is now widely accepted that early surgical intervention is indicated for all patients who present with rapid progressive neurological impairment [4]. Moreover, the development of intraoperative neurophysiological monitoring (IONM) of sensory and motor functions and the use of ultrasonic aspirators have been crucial to guide and perform surgical resection as the first line treatment for these challenging lesions [21]. Despite this, surgical therapy can be performed in selected cases only, and several factors should be taken into account when considering it, such as age and performance status of the patient, prognosis, as well as location and severity of the primary tumor, anesthesiologic and surgical risks $[4,8,15,22,23]$. The concept of spinal instability is also critical in the surgical decisionmaking process.

In order to predict spinal instability due to neoplastic lesions, Fisher et al., developed a classification system based on patient symptoms and radiographic criteria of the spine [24]. The Spine Instability Neoplastic Score (SINS) may guide surgeons in assessing the key components of spinal instability and may become a prognostic tool together with patient-specific factors [24]. Another classification system was proposed by Tokuhashi et al. in 1990, potentially useful for preoperative evaluation of metastatic spine tumors prognosis [25]. In 2005, the authors revised the system [26] by correlating the following parameters with survival period: general medical conditions, number of extraspinal bone metastases, number of metastases in the vertebral body, metastases to the major internal organs such as lungs and brain, primary site of cancer and severity of spinal cord impairment. Each parameter ranged from 0 to 5 points, and the total score was 15 points. Conservative treatment or palliative procedures were indicated with a total score of 8 or less or those with multiple vertebral metastases, while excisional procedures were suggested in patients who scored 12 or more, or in those with a total score of 9 to 11 and with metastases in a single vertebra [26]. Despite most of ISCMs are well encapsulated and can be safely removed, Gasser et al. [9] noted that tumor histology also influenced the extent of resection and radicality. They found that poorly differentiated carcinomas and sarcomas were difficult to dissect due to the lack of a clear border between the tumor and the spinal cord, as well as an increased likelihood of clinical deterioration after lesion resection in these patients [9].

According to the authors, complete surgical removal should be reserved for those cases where a clear plane of cleavage allows a safe microsurgical dissection of the lesion [9], whereas biopsy, decompressive laminectomy and adjuvant therapy may prove as valuable alternatives to radical resection in the remaining cases [9]. Radiation therapy and chemotherapy are considered for adjuvant treatment for ISCMs patients. Post-operative conventional external beam radiation therapy has been the standard of care [27], especially considered for radio-sensible cancers such as small cell lung and breast carcinomas, and lymphomas. However, technical improvements have allowed for the application of the stereotactic body radiotherapy (SBRT) to the spine, which delivers several fold greater biological effective doses [27]. Outcome data show higher rates of local control and pain control for patients treated by SBRT, and suggest better efficacy than with conventional palliative radiotherapy $[28,29]$, with vertebral compression fractures being the most concerning adverse event, usually observed in $10-15 \%$ of patients [27]. Adjuvant radiation therapy (total dose 30-40 Gy) may be also useful for residual tumor after surgery and recurrent tumor, but controversy exists regarding this treatment [30]. Boström et al. [31] reported 70 cases of intramedullary spinal cord tumors, among which 8 were represented by carcinoma metastases. Adjuvant therapy in the form of radiotherapy was administered in 5 out of 8 cases, while 2 patients received adjuvant chemotherapy and the remaining one underwent palliative chemotherapy alone [31].

Although standards of treatment have improved in these years, prognosis of ISCMs remains poor, and median survival varies from 9.4 months when patients have undergone surgery to 5 months when conservative treatment only has been adopted [4].So far, few papers have been published about surgical resection of ISCMs. Hrabalek [12] reported 42 references of 87 ISCM surgically treated up to January 2009, found through a biomedical database search [12]; they only found 13 references of 27 cases with diagnosed and treated ISCMs of breast carcinoma. In 2013, Rostami et al. [32] found a total of 36 papers, among which 19 were single case reports or small case series exclusive of breast cancer metastases [33].

Breast cancer shows a high propensity to spread to the central nervous system (CNS) during its course [32]. Nonetheless, ISCMs are probably the rarest type of CNS involvement from this kind of cancer, representing $8.5 \%$ of all cases of CNS metastases [15], with only sporadic cases being reported in the literature. Lung carcinoma represents the second most common non-CNS malignancy being source of ISCMs as the result of rapidly progressing systemic cancer, accounting for $59 \%$ of all cases. Other tumor primaries include kidney and melanoma (6\% for both), colorectal (5\%), Hodgkin's disease (4\%), and other primary tumors (9\%), whereas the thoracic levels are the most common locations of ISCMs [34]. Rarely, the primary source of ISCMs is not found despite histopathological 
examination. Raco A., et al. reported a case of cervicothoracic intramedullary metastasis in a 22-year-old female patient, in whom the primary tumor was not detected [35]. Of all spinal tumor's locations, extradural is the most common (55\%), primarily in the vertebral body and the epidural fat [14]. ISCMs usually present as solitary lesions. In an Institutional radiological case series of 70 metastasis diagnosed by MRI in 49 patients, Rykken et al. [19] reported solitary metastases in 39 (80\%) cases and multiple ISCMs in 10 (20\% of patients).

In our literature review, we found only one case report about a surgically treated double intramedullary metastasis in patient suffering from breast cancer, but the repetitive lesions occurred separately, each of which as a solitary lesion [36]. In contrast, we reported the treatment of two different intramedullary metastases occurring at the same time. These lesions were very close, resembling twins, and this is the reason why we called them "twins' tumors". To our knowledge, this is the first case report describing such phenomenon. In addition, the masses were very different in terms of consistency, thus requiring two different surgical strategies to obtain radical resection. The first, superior mass, was soft and well circumscribed and excision was performed by en bloc resection without traumatic manipulation of spinal cord parenchyma. In this first step, neurophysiological monitoring of D-Wave, MEPs and SSEPs remained unchanged. The second mass, that developed in a lower position, was harder than the first one, and adherent to spinal cord parenchyma. Since en bloc or piecemeal resection was not feasible, we needed to use an ultrasound aspirator to achieve a radical resection. During this second surgical step, MEPs transiently disappeared, while the D-Wave remained stable throughout the procedure. Despite the intraoperative MEPs loss, the neurological status of the patient improved after surgery.

\section{Conclusion}

This is the first case described in literature where two different intramedullary metastases from breast cancer occurred simultaneously, in absence of any other systemic repetitive lesion. We reported two different modalities of surgical resection of ISCMs, with and without ultrasound aspirator, used during the same operation, with side effects of this surgical tool on spinal cord parenchyma, as demonstrated by IONM. Our report highlights the importance of using multimodal IONM in order to improve the safety of the surgical procedure, preserving neuronal structures and achieving an optimal postoperative outcome [37]. The use of ultrasound aspirator should be limited to those tumors whose firm consistency prevents other surgical strategies to be applied.

\section{Conflict of interest}

We have no disclosure to make that qualifies as a conflict of interest.

\section{References}

1. Watanabe M, Nomura T, Toh E, Sato M, Mochida J (2006) Intramedullary spinal cord metastasis: a clinical and imaging study of seven patients. J Spinal Disord Tech 19(1): 43-47.

2. Grem JL, Burgess J, Trump DL (1985) Clinical features and natural history of intramedullary spinal cord metastasis. Cancer 56(9): 23052314

3. Jellinger K, Kothbauer P, Sunder-Plassmann E, Weiss R (1979) Intramedullary spinal cord metastases. J Neurol 220(1): 31-41.

4. Kalayci M, Cağavi F, Gül S, Yenidünya S, Açikgöz B (2004) Intramedullary spinal cord metastases: diagnosis and treatment - an illustrated review. Acta Neurochir (Wien) 146(12): 1347-1354; discussion 1354. Epub 2004 Nov 8

5. Dam-Hieu P, Seizeur R, Mineo JF, Metges JP, Meriot P, et al. (2009) Retrospective study of 19 patients with intramedullary spinal cord metastasis. Clin Neurol Neurosurg 111(1): 10-17.

6. Edelson RN, Deck MD, Posner JB (1972) Intramedullary spinal cord metastases. Clinical and radiographic findings in nine cases Neurology 22: $1222-1231$.

7. Schiff D, O'Neill BP (1996) Intramedullary spinal cord metastases: clinical features and treatment outcome. Neurology 47: 906-912.

8. Findlay JM, Bernstein M, Vanderlinden RG, Resch L (1987) Microsurgical resection of solitary intramedullary spinal cord metastases. Neurosurgery 21(6): 911-915.

9. Gasser T, Sandalcioglu IE, El Hamalawi B, van de Nes JA, Stolke D, et al. (2005) Surgical treatment of intramedullary spinal cord metastases of systemic cancer: functional outcome and prognosis. J Neurooncol. 2005 Jun;73(2):163-168.

10. Connolly ES, Winfree CJ, Mc Cormick PC, Cruz M, Stein BM (1996) Intramedullary spinal cord metastasis: report of three cases and review of the literature. Surg Neurol 46: 329-337; discussion 337-338.

11. Payer S, Mende KC, Westphal M, Eicker SO (2015) Intramedullary spinal cord metastases: an increasingly common diagnosis. Neurosurg Focus 39(2): E15.

12. Hrabalek L (2010) Intramedullary spinal cord metastases: review of the literature. Biomed Pap Med Fac Univ Palacky Olomouc Czech Repub 154 (2): $117-122$.

13. Potti A, Abdel-Raheem M, Levitt R, Schell DA, Mehdi SA (2001) Intramedullary spinal cord metastases (ISCM) and non-small cell lung carcinoma (NSCLC): clinical patterns, diagnosis and therapeutic considerations. Lung Cancer 31(2-3):319-323.

14. Costigan DA, Winkelman MD (1985) Intramedullary spinal cord metastasis. A clinicopathological study of 13 cases. J Neurosurg 62(2): 227-233.

15. Ogino M, Ueda R, Nakatsukasa M, Murase I (2002) Successful removal of solitary intramedullary spinal cord metastasis from colon cancer. Clin Neurol Neurosurg 104(2): 152-156.

16. Sutter B, Arthur A, Laurent J, Chadduck J, Friehs G, et al. (1998) Treatment options and time course for intramedullary spinal cord metastasis. Report of three cases and review of the literature. Neurosurg Focus 4(5): e3.

17. Kalita 0 (2011) Current insights into surgery for intramedullary spinal cord metastases: a literature review. Int J Surg Oncol 2011:989506.

18. Dunne JW, Harper CG, Pamphlett R (1986) Intramedullary spinal cord metastases: a clinical and pathological study of nine cases. Q J Med 61(235): 1003-1020. 
19. Rykken JB, Diehn FE, Hunt CH, Schwartz KM, Eckel LJ, et al. (2013) Intramedullary Spinal Cord Metastases: MRI and Relevant clinical Features from 13-Year Institutional Case Series AJNR Am J Neuroradiol 34: 2043-49.

20. Decker RE, Sundrani S, Citron ML, Herrschaft DS (1987) Intramedullary spinal cord metastases treated by complete resection of tumor prior to radiotherapy and chemotherapy. Case report and review. Spine (Phila Pa 1976) 12(4): 393-395.

21. Jörg Klekamp (2013) Treatment of intramedullary tumors: analysis of surgical morbidity and long-term results. J Neurosurg Spine 19: 12-26.

22. Schijns OE, Kurt E, Wessels P, Luijckx GJ, Beuls EA (2000) Intramedullary spinal cord metastasis as a first manifestation of a renal cell carcinoma: report of a case and review of the literature. Clin Neurol Neurosurg 102(4): 249-254

23. Stranjalis G, Torrens MJ (1993) Successful removal of intramedullary spinal cord metastasis: case report. Br J Neurosurg 7(2): 193-195.

24. Fisher CG, DiPaola CP, Ryken TC, Bilsky MH, Shaffrey CI, et al. (2010) A novel classification system for spinal instability in neoplastic disease: an evidence-based approach and expert consensus from the Spine Oncology Study Group. Spine (Phila Pa 1976) 35(22): E1221-9.

25. Tokuhashi Y, Matsuzaki H, Toriyama S, Kawano H, Ohsaka S (1990) Scoring system for the preoperative evaluation of metastatic spine tumor prognosis. Spine (Phila Pa 1976) 15(11): 1110-1113.

26. Tokuhashi Y, Matsuzaki H, Oda H, Oshima M, Ryu J (2005) A revised scoring system for preoperative evaluation of metastatic spine tumor prognosis. Spine (Phila Pa 1976) 30(19): 2186-2191.

27. Alghamdi M, Tseng CL, Myrehaug S, Maralani P, Heyn C, et al. (2017) Postoperative stereotactic body radiotherapy for spinal metastases. Chin Clin Oncol 6(Suppl 2): S18.

28. Sahgal A, Bilsky M, Chang EL, Ma L, Yamada Y, et al. (2011) Stereotactic body radiotherapy for spinal metastases: current status, with a focus on its application in the postoperative patient. J Neurosurg Spine 14(2): $151-66$

ISSN: 2574-1241

DOI: 10.26717/BJSTR.2020.29.004749

Pizzuti V. Biomed J Sci \& Tech Res

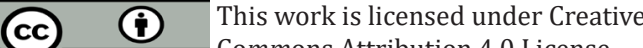

Submission Link: https://biomedres.us/submit-manuscript.php
29. Sahgal A, Larson DA, Chang EL (2008) Stereotactic body radiosurgery for spinal metastases: a critical review. Int J Radiat Oncol Biol Phys 71(3): 652-665.

30. Isaacson SR (2000) Radiation therapy and the management of intramedullary spinal cord tumors. J Neurooncol 47(3): 231-238.

31. Boström A, Kanther NC, Grote A, Boström J (2014) Management and outcome in adult intramedullary spinal cord tumours: a 20-year single institution experience. BMC Res Notes 7: 908.

32. Rostami R, Safarpour D, Tavassoli FA, Jabbari (2013) Intramedullary metastases in breast cancer- a comprehensive literature review. Journal of the Neurological Sciences 332(1): 16-20.

33. Kosmas C, Koumpou M, Nikolaou M, Katselis J, Soukouli G, et al. (2005) Intramedullary spinal cord metastases in breast cancer: report of four cases and review of the literature. J Neurooncol 71(1): 67-72.

34. Schwechheimer K, Lemminger JM (1985) Intramedullary metastases: report of 4 cases and review of the literature. Clin Neuropathol 4(1): 2837.

35. Raco A, Delfini R, Salvati M, Innocenzi G, Ciappetta P (1992) Intramedullary metastasis of unknown origin: a case report. Neurosurg Rev 15(2): 135-138.

36. Hyun Chul Choi, Do Heum Yoon, Seung Chul Kim, Ki Hong Cho, Sang Hyun Kim (2010) Two Separate Episodes of Intramedullary Spinal Cord Metastasis in a Single Patient with Breast Cancer J Korean Neurosurg Soc 48: 162-165.

37. Antonino Scibilia, Carmen Terranova, Vincenzo Rizzo, Giovanni Raffa, Adolfo Morelli, et al. (2016) Intraoperative neurophysiological mapping and monitoring in spinal tumor surgery: sirens or indispensable tools? Neurosurgical Focus 41(2): E18.

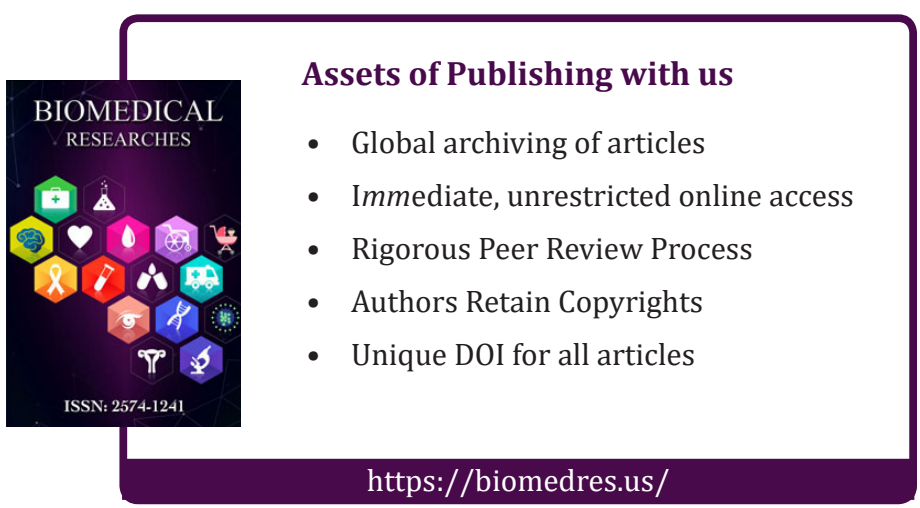

